\title{
$\Rightarrow$ INFLAMMATION
}

\section{Activated protein $\mathrm{C}$ inhibits inflammasome activation in IRI}

Activated protein $\mathrm{C}(\mathrm{aPC})$ protects against ischaemia-reperfusion injury (IRI) by inhibiting activation of the NLRP3 inflammasome, say researchers Berend Isermann and colleagues. Previous studies have shown that aPC has anti-inflammatory and anti-apoptotic effects and can protect against IRI in various organs, but the mechanism was unclear. "As the inflammasome constitutes an important mechanism linking inflammation and cell death, we hypothesized that aPC inhibits inflammasome activation," explains Isermann.

The researchers report that in mice, myocardial IRI induces NLRP3 inflammasome activation prior to induction of apoptosis and the detection of an infarction, suggesting a pathogenic role of the inflammasome. Treatment with aPC before induction of myocardial or renal IRI resulted in reductions in NLRP3 inflammasome activation, apoptosis and tissue damage. These protective effects were abolished in mice that expressed constitutively active NLRP3, confirming that inhibition of inflammasome activation is responsible for aPC-mediated protection in the setting of IRI.

Further studies showed that aPC treatment inhibits NLRP3 inflammasome activation via a mechanism involving inhibition of PAR1 and mTORC1 signalling. Inhibition of PAR1 signalling abolished the protective effect of aPC in myocardial IRI, whereas blocking the anticoagulant function of aPC had no effect. In addition, targeting PAR1 signalling using parmodulin 2 mimicked the protective effects of aPC in IRI.

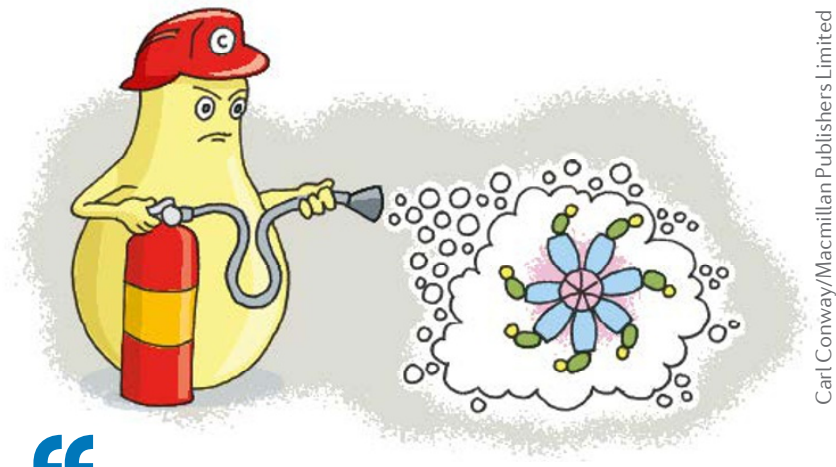

We suspect that perfusion of a donor organ with aPC ... before transplantation would inhibit inflammasome activation and improve organ function The researchers suggest that therapies based on their new findings might be important in the setting of organ transplantation. "We suspect that perfusion of a donor organ with aPC or an aPC-based therapeutic agent before transplantation would inhibit inflammasome activation and improve organ function," comments Isermann.

Ellen F. Carney

ORIGINAL ARTICLE Nazir, S. et al.

Cytoprotective activated protein C averts Nlrp3

inflammasome induced ischemia reperfusion

injury via mTORC1 inhibition. Blood

http://dx.doi.org/10.1182/blood-2017-05-782102

(2017) 